\title{
Las pruebas diagnósticas en la didáctica de la lengua y la literatura ${ }^{1}$
}

\author{
Manuel MARTÍ SÁNCHEZ y M. Teresa PÉREZ TAPIA \\ Universidad de Alcalá (España) \\ Departamento de Filología, Comunicación y Documentación \\ manuel.marti@uah.es; teresa.perezt@uah.es
}

Recibido: junio 2012

Aceptado: enero 2013

\section{RESUMEN}

En este artículo se presenta una prueba diagnóstica elaborada para la medición de las habilidades lingüístico-comunicativas en los estudiantes de Secundaria. En la fundamentación teórica de dicha prueba diagnóstica ocupa una posición clave la conciencia metalingüística. Palabras clave: habilidades lingüístico-comunicativas, didáctica de la lengua y la literatura, evaluación del lenguaje.

Les tests diagnostic dans la didactique des langues et des littératures

\section{RÉSUMÉ}

Cet article présente un test diagnostic mis au point pour mesurer les habiletés de communication chez les élèves du collège. Dans les fondements théoriques de ce test diagnostic, la conscience métalinguistique joue un rôle clé.

Mots-clés: habiletés de communication, didactique de la Langue et de la Littérature, évaluation du langage.

\section{Diagnostic tests in Language and Literature Education}

\begin{abstract}
In this paper presents a diagnostic test developed to measure language skills in Middle School students. On the theoretical basis of the diagnostic test metalinguistic, metalinguistic consciousness has a key role.
\end{abstract}

Key words: language skills, language and literature teaching, language testing.

${ }^{1}$ Este artículo forma parte de las actividades desarrolladas en el Proyecto de investigación "La atención a la forma lingüística en la materia de Lengua y Literatura en Secundaria" (Ref.: UAH2011/HUM-005). Agradecemos los muy acertados comentarios a la primera versión de este artículo realizados por el profesor Díaz-Corralejo. 


\section{SUMARIO}

1. Presentación. 2. Competencia comunicativa y habilidades lingüístico-comunicativas. 2.1. Presentación. 2.2. Estructura. 2.3. Adquisición/ aprendizaje de las habilidades lingüísticocomunicativas. 3. Diseño de la prueba diagnóstica inicial. 3.1. Hipótesis. 3.2. Estructura. 3.3. Evaluación. 4. Conclusiones

\section{PRESENTACIÓN}

El cúmulo de factores que intervienen en el doble proceso de enseñanza/ aprendizaje y su aparición irregular convierten dicho proceso en una actividad bastante incontrolable. Steiner y Ladjali (2005 [2003]) son bastante concluyentes:

La libertad individual y una suma muy amplia de variables y circunstancias convierten la enseñanza en una actividad bastante misteriosa, donde nunca pueden predecirse con seguridad los resultados.

Que esto es así lo manifiesta un hecho tan cotidiano como la facilidad con que todo lo planificado se estrella con la desordenada realidad del aula, de ahí la resistencia de los docentes ante una construcción teórica cada vez más técnica y burocratizada.

Tal evidencia debe hacer bastante humilde al investigador en Didáctica de la lengua y la literatura (DLyL) y convencerlo de la imposibilidad de hacer predicciones seguras, al modo de las ciencias fuertes y duras. Sin embargo, el baño de realidad no debe ahogar su legítima aspiración a mejorar la realidad del aula, por muy desalentadora que pueda resultar esta. En esta dialéctica realidad/deseo, se mueve la DLyL, que ha de investigar

cómo se construye el conocimiento verbal, qué relación se establece entre modelos de enseñanza y procesos de aprendizaje, qué incidencia tienen en la interacción los diferentes tipos de materiales, cómo se pueden adecuar las programaciones... (Camps y Ruiz Bikandi 2011: 27).

Sin este análisis no hay propuestas sólidas que sirvan al trabajo predictivo en el aula dentro de la investigación de acción que quiere desarrollarse (Camps 2012: 33). Sin este análisis se cae en el teoricismo estéril que siempre acecha, a pesar de que se invoquen como talismán el enfoque comunicativo, la competencia comunicativa, las habilidades verbales, el construccionismo...

Entre los cometidos de la DLyL está la elaboración de pruebas diagnósticas que proporcionen un conocimiento de la situación de los estudiantes con los que va a trabajarse. Tal conocimiento es imprescindible para calcular rigurosamente la mejora experimentada en estos, el papel en ella de la instrucción suministrada y, en consecuencia, articular medidas correctoras.

En este artículo va a describirse una prueba diagnóstica. Puesto que su destino lo constituyen las habilidades lingüístico-comunicativas, eje del currículo oficial de la materia de Lengua y Literatura en Secundaria, antes haremos una esquemática aproximación a la naturaleza y los factores del desarrollo de estas habilidades. Esta fundamentación ayudará a orientar la prueba diagnóstica.

La prueba diagnóstica se ha elaborado para los estudiantes de Secundaria de dos centros públicos españoles de Secundaria, el IES "Liceo Caracense" (Guadalajara) 
y el IES "Albalat" (Navalmoral de la Mata, Cáceres). Sin embargo, no es una prueba ad hoc, su campo de aplicación quiere ser general, por esto, tenemos pensado utilizarla también en algunos centros públicos rurales nicaragüenses como coda final del Proyecto de Cooperación Interuniversitaria "Bachilleres geográficamente alejados para su integración universitaria en Nicaragua", en el que hemos participado $^{2}$.

Para alcanzar esta validez, la prueba diagnóstica ha de ofrecer datos fiables de las habilidades verbales de cualquier grupo de estudiantes de la asignatura de Lengua y Literatura en Secundaria haciéndolas visibles en sus aspectos más relevantes para la DLyL. Esta exigencia sustantiva se acompaña de otra de índole formal o metodológica, consistente en que la prueba se ajuste a la realidad del aula, de modo que sea viable en el sentido de sencilla de aplicar y cuente con criterios de evaluación objetivos, independientes de quien mida los resultados.

\section{COMPETENCIA COMUNICATIVA Y HABILIDADES LINGÜÍSTICO- COMUNICATIVAS}

\subsection{Presentación}

Desde el lejano artículo de Hymes de 1966 sobre la competencia comunicativa (renombrada posteriormente competencia en comunicación lingüistica o competencias comunicativas de la lengua) ${ }^{3}$, mucho se ha escrito sobre ella, particularmente en la enseñanza de lenguas (Pérez Estévez y Zayas 2007; Coste et al. 2012), como ya previó Hymes en el punto 6 del posfacio de 1982 a su monografía de 1973 (Hymes1984).

Para Hymes (1972: 60), la competencia comunicativa es ese conocimiento acerca de "cuándo hablar, cuándo no, así como de qué hablar, con quién, de qué manera". Por la competencia comunicativa, "un niño es capaz de alcanzar un repertorio de actos verbales, tomar parte en acontecimientos verbales y evaluar su logro por otros". A partir de esta idea inicial, como suele suceder, la competencia comunicativa se ha desarrollado y multiplicado sus componentes (Hymes 1985: 16-19), a la

\footnotetext{
${ }^{2}$ En dos ediciones, este proyecto se ha desarrollado de 2009 a 2011, cuyo informe final se presentó el 8 de febrero de 2012 en León (Nicaragua). En tal informe se puso de manifiesto, entre otras necesidades, contar con sistemas de evaluación más eficientes. Esta necesidad se dejó sentir en la prueba de acceso a la Universidad, acerca de la que el equipo de español de este proyecto (Manuel Martí, Ana M. Ruiz y Fernando Chicharro) elaboró un informe en 2011 para la UNAM-León (Nicaragua).

${ }^{3}$ La primera es como se la conoce oficialmente en la normativa española de Secundaria; la segunda es como la nombra en el Marco Común Europeo de Referencia (MCER) (Consejo de Europa 2003). Que en el MCER se hable de Competencias es coherente con el número de competencias distinguidas dentro o en relación con la competencia comunicativa (Hymes 1985: 16-17).
} 
par que se han puesto de relieve algunas inconsistencias (Acard 2005; Coste et al. 2012).

La competencia comunicativa aparece en el currículo oficial de la Secundaria española como las habilidades lingüístico-comunicativas ${ }^{4}$, a las que se subordinan la educación literaria (que tiene como meta la construcción del lector literario) y el conocimiento de la lengua, bloque que se reserva a la enseñanza metalingüística más técnica:

El eje del currículo son las habilidades y estrategias para hablar, escribir, escuchar y leer en ámbitos significativos de la actividad social. Estos aprendizajes se recogen en tres de los bloques de contenidos del currículo: 1. Hablar, escuchar y conversar. 2. Leer y escribir. 3. Educación literaria. En relación con ellos, el bloque 4, Conocimiento de la lengua, reúne los contenidos que se refieren a la capacidad de los alumnos para observar el funcionamiento de la lengua y para hablar de ella, así como a los conocimientos explícitos sobre la lengua y sus formas de uso (REAL DECRETO 1631/2006, de 29 de diciembre, por el que se establecen las enseñanzas mínimas correspondientes a la Educación Secundaria Obligatoria) (REAL DECRETO 1631/2006, de 29 de diciembre, por el que se establecen las enseñanzas mínimas correspondientes a la Educación Secundaria Obligatoria, BOE núm. 5, viernes 5 de Enero de 2007).

Estas palabras dejan claro que en la enseñanza de la lengua materna, como antes en la de la extranjera, se ha impuesto el enfoque comunicativo. Además, si nos fijamos, en esta cita, como en las palabras anteriores de Hymes, se señala que tanto la competencia comunicativa como las habilidades lingüístico-comunicativas suponen saberes que exceden el puro uso lingüístico apuntando a otros sistemas cognitivos. De esto hablaremos en $\S 2.3$.

En cualquier caso, no hay duda de que la competencia comunicativa y, aún más, estas habilidades representan un conocimiento de naturaleza procedimental, un saber hacer, pues. Que esto sea así no supone que a ese conocimiento no pueda accederse parcialmente mediante la reflexión y convertirse en declarativo. Aquí radica un factor fundamental en el desarrollo de las habilidades.

\subsection{Estructura}

Existen diversos modelos sobre los componentes de la competencia comunicativa. En el MCER se postulan las siguientes competencias comunicativas de la

${ }^{4}$ Estas habilidades (abilities, skills) funcionan, a veces, como término alternativo al de competencia comunicativa de los que habla Hymes (1985: 16). Lo mismo puede decirse de destrezas o proficiencia (proficiency). Como también los términos son unidades simbólicas, no deberían intercambiarse al modo de los sinónimos perfectos, lo que solo es posible si se emprende una depuración terminológica. En este punto nos interesa especialmente la distinción entre competencia y habilidades o destrezas (Cenoz Iragui 2004; Escandell 2006). 
lengua: LINGÜÍSTICAS (léxica, gramatical y semántica; fonológica, ortoépica y ortográfica), SOCIOLINGÜÍSTICA y PRAGMÁTICAS (discursiva y funcional).

En los modelos más detallados de estas, las competencias comunicativas de la lengua se entrecruzan con las mencionadas cuatro habilidades lingüísticocomunicativas (expresión oral, expresión escrita, comprensión auditiva y comprensión lectora), a las que puede añadirse la interacción oral ${ }^{5}$.

El éxito alcanzado por la competencia comunicativa la ha hecho crecer a costa de la competencia gramatical y de las otras competencias. La expansión primera choca con las buenas razones argüidas para considerar la competencia gramatical y pragmática $^{6}$ como complementarias (Escandell 2006: 219-231). Sin embargo, el hecho cierto de que en la producción e interpretación intervienen las reglas gramaticales ha conducido a que en la DLyL se incluya la competencia gramatical (o lingüística) en la competencia comunicativa. Así ha sucedido en los distintos modelos de la competencia comunicativa propuestos en esta área (Canale y Swain, Canale, Bachman; Celce-Murcia, Dörnyei y Thurrell; Bachman y Palmer...).

Por otro lado, está la invasión de las otras competencias (aprender a aprender, digital, autonomía persona o cultural).Esta es una consecuencia de la naturaleza de la competencia comunicativa-ya, desde su origen, abierta a lo extralingüístico- y de las subcompetencias comunicativas estratégica e intercultural, claramente multidisciplinares y objeto privilegiado de los estudiosos actuales.

A esos motivos del expansionismo de la competencia comunicativa hay que añadir otros dos factores más extrínsecos. En primer lugar, la triple dimensión del alumno de lenguas que establece el Plan Curricular del Instituto Cervantes y que define los objetivos generales de este:

a) El alumno como agente social que interactúa mediante realizaciones, que no son únicamente de lenguaje, en unas circunstancias y en un contexto dados, dentro de una acción particular y compleja,[en la] que la comprensión del otro es la base de la comunicación (Díaz-Corralejo 2002-2003: 34).

b) El alumno como hablante intercultural, que ha de ser capaz de identificar los aspectos relevantes de la nueva cultura a la que accede a través del español y establecer puentes entre la cultura de origen y la de los países hispanohablantes.

c) El alumno como aprendiente autónomo, que ha de hacerse gradualmente responsable de su propio proceso de aprendizaje, con autonomía suficiente para continuar avanzando en su conocimiento del español más allá del propio currículo, en un proceso que pueda prolongarse a lo largo de toda la vida $<$ http://cvc.cervantes.es/ensenanza/biblioteca_ele/plan_curricular/introduccion.htm>.

${ }^{5}$ En Díaz-Corralejo (2002-2003: 34-38) puede encontrarse una descripción muy precisa del MCER, mucho mayor de la que aparece en estas páginas.

${ }^{6}$ Este es el nombre que le da Chomsky a la competencia comunicativa de Hymes. Para Chomsky (1980: 225), la competencia pragmática es el "conocimiento de las condiciones y manera de usar apropiadamente, de acuerdo con las distintas intenciones". 
En segundo lugar, hay que considerar la función pedagógica de la lengua, por la que esta funciona como una herramienta de aprendizaje (Tolchinsky 2011: 24). Estos factores y la propia identidad de la competencia comunicativa llevan a concluir que la competencia comunicativa implica los sistemas cognitivos de los que dependen habilidades cognitivas humanas como la clasificación, el procesamiento secuencial y la planificación (Grupo Las "cinco gracias" 2011), las normas culturales y el propio código (Escandell 2006).

\subsection{Adquisición/ aprendizaje de las habilidades lingüístico-comunicativas}

Por lo que acaba de decirse, el objetivo de una competencia comunicativa madura, representada por las mencionadas habilidades, no puede basarse solo en el desarrollo único de la competencia lingüística. Son necesarias también las habilidades cognitivas y culturales, así como los respectivos sistemas que las sustentan.

Si nuestro conocimiento avanza mediante la imitación y el juego, la adquisición y el aprendizaje de la lengua materna -el español en el caso de la mayoría de los estudiantes de la Secundaria española- puede decirse que se produce usándola y reflexionando sobre ella (Tolchinsky 2011: 34), justamente, "para resolver situaciones de uso" (Tolchinsky 2011: 35). Desde esta posición, puede decirse que

la adquisición es una cuestión de muestreo, lo que supone una captación de las normas de la población a partir de la muestra aportada por la limitada experiencia del aprendiz, percibida dentro de los límites y posibilidades de su aparato cognitivo, de su corporeización humana y de la dinámica de la interacción social (Grupo Las "cinco gracias" 2011)

En este proceso tres entidades destacan: el lenguaje, la memoria y la conciencia. Las tres se realimentan mutuamente y las tres han de evolucionar para que las habilidades lingüístico-comunicativas lo hagan (cfr. Sabah 1995). Estas no alcanzan exitosamente los fines del hablar, la representación del pensamiento y la interacción con los otros, sin el soporte de la lengua, la memoria y la conciencia.

Este proceso -inconsciente y consciente, espontáneo y voluntario- evoluciona naturalmente. Sin embargo, todos reconocen que es imposible o al menos muy difícil lograr "un nivel alto de competencia lingüística sin que intervengan procesos deliberados de reflexión sobre lo que sabemos de la lengua" (Tolchinsky 2011: 37. La cursiva en el original) ${ }^{7}$.Superar la barrera del desarrollo natural es, en consecuencia, la tarea de la educación. Tal planteamiento ha colocado en un papel central la reflexión o la conciencia metalingüística. Así las cosas, se entiende la

\footnotetext{
${ }^{7}$ Que así suceda, no puede extrañar dado que el desarrollo intelectual puede entenderse como el progreso desde una cognición menos reflexiva, fundamentalmente procedimental, hacia una mayor capacidad de autorreflexión y explicitación llevada a cabo gracias justamente al lenguaje (Karmiloff-Smith 1994).
} 
teorización actual sobre la didáctica de la lengua cada vez más sensible al papel de la metacognición en el desarrollo cognitivo (Perinat 2003: 280) ${ }^{8}$.Este hecho ha sido la causa del peso de las cuestiones metalingüísticas y de las explicaciones de los alumnos en la prueba diagnóstica que presentamos.

\section{DISEÑO DE LA PRUEBA DIAGNÓSTICA INICIAL}

\subsection{Hipótesis}

Al término de la Educación Primaria (6-11 años), los estudiantes han debido adquirir una competencia comunicativa básicamente procedimental que se manifiesta en cierto grado de manejo en las habilidades lingüísticas orales, adquiridas en su mayor parte de forma espontánea, y escritas, aprendidas con la mediación del adulto (padres y maestros). En la Primaria puede haberse iniciado un acercamiento al conocimiento metalingüístico, si bien en pocas ocasiones se tratará de una auténtica autorreflexión sobre la lengua. Por ejemplo, el aprendiz estará en condiciones de identificar un verbo en una oración e incluso habrá memorizado la conjugación verbal, aunque difícilmente comprenderá los matices aspectuales o modales que pautan la aparición de las variantes flexivas verbales en un texto realmente producido ni sabrá manejar estas - de forma consciente- para producir determinados efectos en una composición escrita propia.

En el proceso intelectual de la redescripción representacional ${ }^{9}$ (Karmiloff-Smith 1994), al término de la Primaria se ha iniciado la fase metalingüística, sentando las bases para el desarrollo de la conciencia explícita del sistema de la lengua y su uso. Es en la Secundaria cuando en rigor se comienza el proceso sistemático de reflexión sobre la lengua con el propósito de despertar y desarrollar, a lo largo de los cuatro años de la parte obligatoria de la etapa, un saber consciente y declarativo sobre los mecanismos lingüísticos. Puesto que se trata de un proceso que requiere la mediación del docente, cabe formularse una serie de preguntas cuyas respuestas orientarán al profesor sobre el método y la eficacia de la instrucción lingüística:

- ¿Qué grado de conciencia metalingüística implícita presentan los alumnos en el primer curso de Secundaria?

- ¿La reflexión sobre la lengua y su uso en el contexto escolar incrementan el grado de conciencia metalingüística y la capacidad de verbalización de los conocimientos y los fenómenos lingüísticos?

\footnotetext{
${ }^{8}$ Relacionada con la metacognición está la competencia estratégica, referida a la capacidad del hablante para resolver los distintos problemas sirviéndose de los distintos recursos de que dispone (Sanjuán Bornay 2010).

${ }^{9}$ Proceso por el que el conocimiento implícito e inconsciente va pasando a ser explícito y consciente.
} 
- En caso de existir un progreso demostrable, ¿es paulatino y va incrementándose según el alumno avanza en los cursos de la etapa?

- ¿Existe relación entre la capacidad de verbalización del conocimiento metalingüístico y el desarrollo de las habilidades comunicativas?

- ¿La metodología docente influye en el progreso de los estudiantes?

La respuesta a estas cuestiones puede obtenerse mediante la realización de una prueba diagnóstica que proporcione datos que permitan extraer conclusiones a este respecto. Esta prueba diagnóstica debe satisfacer tanto los interrogantes relativos al grado de conciencia metalingüística y de verbalización de la misma como a los referidos al desarrollo de las habilidades lingüísticas. De ahí que la prueba que aquí se propone se haya estructurado en dos partes: un cuestionario metalingüístico y una propuesta doble de composición escrita (ver, abajo, § 3.2). Para poder extraer datos sobre los efectos de la instrucción en la asignatura de Lengua castellana y literatura y sobre el grado de progreso, la prueba diagnóstica se lleva a cabo por primera vez simultáneamente en los cuatro cursos de la Secundaria Obligatoria. Después, tras la aplicación de un programa de intervención se realizarán pruebas similares que permitan contrastar los nuevos datos con los obtenidos en el proceso diagnóstico.

La hipótesis de trabajo es que el desarrollo de la capacidad de reflexión lingüística incrementa la conciencia metalingüística y declarativa lo que, a su vez, se manifiesta en la creciente destreza del estudiante en el manejo de las habilidades comunicativas, es decir, en su competencia comunicativa.

\subsection{Estructura}

Cuestionario para medir la conciencia metalingüística

Dadas las condiciones de aplicación de la prueba diagnóstica, el cuestionario que se presenta a los estudiantes ha de satisfacer las necesidades del investigador sin presentar un nivel de complejidad excesivo para así resultar accesible para los alumnos más jóvenes.

Cuestionario para medir la conciencia metalingüística

Responde a estas cuestiones:

1. Un enunciado ambiguo es aquel que tiene más de un sentido. Señala y explica la ambigüedad del siguiente enunciado: Estamos a cinco grados bajo cero y llegaremos a once.

2. Un enunciado ambiguo es aquel que tiene más de un sentido. Señala y explica la ambigüedad del siguiente enunciado: Ha acabado tirando la toalla.

3. ¿Cuántos tipos de porqué existen?

4. ¿Qué ejemplo te parece más correcto?, ¿por qué?

a) Dale la noticia de la derrota de nuestro equipo a mis padres.

b) Dales la noticia de la derrota de nuestro equipo a mis padres.

5. ¿Qué clase de palabra te parece que es FUERA? 
6. ¿Qué prefieres que te suceda?
a) Por poco apruebo.
b) Apruebo por poco.
c) Casi no apruebo.
d) Casi apruebo.

7. ¿Qué prefieres que digan de ti?
a) Es lo mejor de la clase.
b) Es de lo mejor de la clase.

El cuestionario permite extraer información del grado de conciencia metalingüística sobre los diferentes niveles de la lengua y su uso: categorías gramaticales (ítems 3 y 5), flexión verbal (ítem 5), concordancia (ítem 4), orden y naturaleza de la modificación (ítem 6), semántica oracional y relación con la situación comunicativa (ítems 1, 2 y 7), etc. En la explicación de la respuesta a cada ítem, es posible observar, además, el manejo de la terminología lingüística (ausente/presente, acertada/desacertada). Por ejemplo, en ítem 2 podría explicarse la causa de la ambigüedad utilizando o no el término locución verbal.

\section{Composición escrita}

La segunda parte de la prueba diagnóstica consiste en dos ejercicios de composición escrita elaborados por los estudiantes de forma individual, en clase y con un tiempo limitado (45 minutos como máximo). La capacidad de expresarse por escrito implica ya ciertos conocimientos lingüísticos así como una mayor conciencia metalingüística (Halliday 1989). "La escritura es esencial para la conciencia lingüística" (Olson 1998: 293) y "realiza el lenguaje en su totalidad" (Barthes 1987: 18).

El primer ejercicio que se propone es la composición de un texto narrativo a partir de una consigna dada y el segundo, algo más libre y complejo, consiste en la escritura de un texto argumentativo en el que el alumno debe justificar su preferencia por uno de dos tipos de personajes fantásticos. Ambas composiciones permitirán observar y registrar las habilidades y microhabilidades lingüísticas de los estudiantes en cuanto a ortografía, empleo de los signos de puntuación, destreza en el uso de recursos morfosintácticos, madurez sintáctica (Hunt 1965), riqueza léxica individual, organización textual y atención a las propiedades macroestructurales derivadas de la tipología del texto.

Escribe un texto narrativo desarrollando la consigna que se propone. Ten en cuenta:

a) Extensión mínima 150 palabras; extensión máxima 200 palabras (sin contar las palabras de la consigna).

b) Puedes elegir para tu relato la formulación de la consigan que prefieras.

\section{CONSIGNA}


Cuando me/te/se desperté/despertaste/despertó, mi/tu/su/ imagen en el espejo me/te/le produjo un profundo espanto.

Escribe un texto argumentativo sobre el tema: ¿Vampiros o licántropos?

Ten en cuenta:

- Extensión mínima 150 palabras; extensión máxima 200 palabras (sin contar las palabras de la consigna).

La redacción de dos tipos de textos diferentes permitirá observar si existen diferencias objetivas entre la composición de la narración, que exige mayor creatividad y a cuyos modelos orales y escritos han estado expuestos los alumnos en su vida académica y personal, y la composición de la argumentación, en la que la experiencia es por fuerza más limitada y exige un mayor grado de capacidad de razonamiento.

\subsection{Evaluación}

El sistema de evaluación de la prueba diagnóstica así como de las que se vayan aplicando como control del progreso debe ser objetivo a fin de garantizar la homogeneidad de criterio para todos los evaluadores y la validez de los datos obtenidos.

En el cuestionario de conciencia metalingüística, cada ítem se valora empleando una escala de $0-1-2$, de modo que el valor 0 se otorgará a respuestas incorrectas que evidencian un grado mínimo o nulo en la reflexión y conciencia metalingüística-, el valor 1 para respuestas que aporten una explicación del fenómeno lingüístico, aunque sin una verbalización técnica del mismo y el valor 2 para las respuestas que ofrezca una explicación correcta y razonada lingüísticamente. Así pues, la puntuación obtenida por cada estudiante en el cuestionario se encontrará comprendida en la horquilla $0-14$.

La evaluación de los ejercicios de composición escrita ha de cumplir los mismos criterios de rigor y objetividad. Para ello se aplican herramientas de medición objetivas ${ }^{10}$ para cada uno de los aspectos lingüísticos que van a ser evaluados.

1. El estudio de la corrección ortográfica se realiza empleando un documento de registro utilizado para las evaluaciones diagnósticas del sistema educativo en la etapa obligatoria ${ }^{11}$. En él, se hace constar el tipo de error cometido por cada uno de los alumnos.

2. El grado de madurez alcanzado en el uso de la puntuación se registrará siguiendo el modelo propuesto por Halliday y citado por Cassany (1993) que

\footnotetext{
${ }^{10}$ Algunas de ellas ya fueron aplicadas en una investigación anterior (Pérez Tapia 2009), en la que pudo comprobarse su eficacia e idoneidad.

${ }^{11}$ Informes del Instituto Nacional de Calidad Educativa (INCE).
} 
permite, debido a la jerarquización en cuanto a la dificultad que entraña su uso, evaluar el nivel de destreza y conocimiento de los estudiantes a la hora de puntuar su texto y establecer así de forma clara (si son altos) o confusa (si son bajos) las relaciones sintácticas pretendidas.

3. Para el análisis de la destreza en el uso de recursos morfosintácticos se procede a una clasificación completa de todas las palabras que son empleadas por cada alumno. En este caso, el criterio para definir palabra es funcional por lo que se incorporan tanto palabras unimembres como locuciones -conjunto de términos funcionalmente equivalentes a una palabra. Las clases que se establecen son: preposiciones, conjunciones, determinantes, pronombres, sustantivos, adjetivos, adverbios, verbos y marcadores discursivos. En los textos de índole narrativa, el protagonismo de los verbos se hace patente no solo en su frecuencia de aparición sino también en la pertinencia del análisis complementario de la flexión verbal y del uso de perífrasis.

4. El grado de madurez sintáctica se mide aplicando los indicadores propuestos por Hunt (cfr. González Ma(r)fud 2009). Se analizan las composiciones escritas de los estudiantes computando el número de unidades terminales ${ }^{12}$ (UT), de cláusulas ${ }^{13}$ (CL) y el número de palabras -aplicando ahora un criterio gráfico- de unidades terminales y cláusulas. Estos datos permiten calcular los promedios de longitud de UT $\left(\mathrm{n}^{\circ}\right.$ de palabras / $\mathrm{n}^{\circ}$ de UT) y CL $\left(\mathrm{n}^{\circ}\right.$ de palabras / $\mathrm{n}^{\mathrm{o}}$ de CL), el promedio de CL por UT ( ${ }^{\circ}$ de cláusulas / $\mathrm{n}^{\mathrm{o}}$ de UT) y la frecuencia relativa de los diferentes tipos de oraciones subordinadas (por ejemplo, $\mathrm{n}^{\circ}$ de subordinadas sustantivas / $\mathrm{n}^{\mathrm{o}}$ de UT). Así serán indicadores de un mayor grado de madurez sintáctica las UT y CL más extensas, y el mayor promedio CL / UT.

5. El estudio del vocabulario activo de cada uno de los estudiantes, permite analizar el índice de riqueza léxica individual. Para ello, se vuelve a realizar un cómputo de palabras, pero esta vez atendiendo al criterio léxico o semántico, sumado al funcional. De ahí que, siguiendo a Humberto López Morales (1984), solo se contabilicen para este fin los sustantivos, adjetivos, verbos y adverbios léxicos, sin tener en cuenta el número de veces que aparecen ni las variantes flexivas que presenten. El número de piezas léxicas diferentes se divide entre el $\mathrm{n}^{\mathrm{o}}$ de palabras del texto escrito por el alumno. El resultado es el índice de riqueza léxica.

\footnotetext{
12 Unidad más corta en que puede dividirse una pieza del discurso sin dejar ningún fragmento de oración como residuo (Hunt: 1965). Oración principal + sus oraciones subordinadas. Se excluyen las de rango principal coordinadas y yuxtapuestas.

${ }^{13}$ Un sujeto o conjunto de sujetos coordinados con un verbo finito o un conjunto finito de verbos coordinados (Hunt: 1965). Oraciones simples, oraciones principales y oraciones subordinadas.
} 
6. La organización textual se mide observando la distribución en párrafos del texto y el uso/ausencia de marcadores discursivos propios de cada tipología textual.

\section{CONCLUSIONES}

Las pruebas diagnósticas en la DLyL son una herramienta imprescindible para evaluar el grado de incidencia del proceso de enseñanza en el de aprendizaje y en el desarrollo de la propia competencia comunicativa de los alumnos. El instrumento de diagnóstico que aquí se propone permite, por su estructuración en dos partes, valorar por separado o en conjunto la eficacia de las enseñanzas relativas a la competencia lingüística -cuestionario de conciencia metalingüística- y de las que persiguen mejorar las habilidades lingüísticas de los estudiantes.

El cuestionario de conciencia metalingüística, cuyos ítems pueden diseñarse en función de la información que pretenda obtenerse, es un instrumento ágil, que permite extraer conclusiones bastante precisas acerca de los conocimientos aprehendidos relativos al funcionamiento del sistema de la lengua en cada uno de sus niveles.

El procedimiento de evaluación de las pruebas de composición escrita, además de poner de manifiesto la incidencia del conocimiento metalingüístico en el desarrollo de la competencia comunicativa, ofrece la ventaja de evidenciar objetivamente -índices de riqueza léxica o madurez sintáctica, por ejemplo- las dificultades o progresos de los aprendices en el manejo de la expresión escrita. La mayor complejidad del procedimiento de evaluación se ve así compensada por la fiabilidad de los resultados.

Las pruebas diagnósticas pueden aplicarse tanto como evaluación inicial, con el propósito de señalar al docente un punto de partida, como en cualquier momento del proceso de enseñanza y aprendizaje, para constatar el progreso del alumnado y la idoneidad de la metodología didáctica elegida.

\section{REFERENCIAS BIBLIOGRÁFICAS}

ACARD, A. (2005): "The 'Communicative Competence' Controversy". Assian EFL Journal, 7.3<http://www.asian-efl-journal.com/September_05_ac.php> [Consulta: marzo 2012].

BARTHES, R. (1987): El susurro del lenguaje. Más allá de la palabra y la escritura. Barcelona: Paidós.

CAMPS, A. (2012): "La investigación en didáctica de la lengua en la encrucijada de muchos caminos". Revista Iberoamericana de Educación 59, 23$41<\mathrm{http}: / /$ www.rieoei.org/rie_revista.php?numero=rie59a01\&titulo=La\%20inve stiga-

ción $\% 20$ en $\% 20$ Didáctica $\% 20 \mathrm{de} \% 201 \mathrm{l} \% 20$ Lengua $\% 20$ en\%201a\%20encrucijada $\% 20 \mathrm{de} \% 20$ muchos\%20caminos> [Consulta: marzo 2013].

CASSANY, D. (1995): La cocina de la escritura. Barcelona: Anagrama. 
CHOMSKY, N. (1980): Rules and Representations. Oxford: Blackwell.

CONSEJO DE EUROPA (2003). Marco común europeo de referencia para las lenguas: aprendizaje, enseñanza, evaluación. Ministerio de Educación, Cultura y Deporte/ Instituto Cervantes/ Anaya http://cvc.cervantes.es/obref/marco [Consulta: marzo 2012].

COSTE, D. et al. (2012): "Hymes et le palimpseste de la compétence de communication. Tours, détours et retours en didactique des langues". Langage et société 139, 103-123.

DÍAZ-CORRALEJO, J. (2002-2003): "Marco Común Europeo de Referencia en el aprendizaje de lenguas extranjeras". Encuentro Revista de investigación e innovación en la clase de idiomas, 13-14, 32-43.

ESCANDELL, $\mathrm{M}^{\mathrm{a}}$ V. (2006): Introducción a la Pragmática. Barcelona: Ariel ${ }^{2}$.

GONZÁLEZ MA(R)FUD, A. M (2009): "La enseñanza de la lengua y la aprehensión del ser cubano". Boletín de la Academia Cubana de la Lengua 12-13-14 $<$ http://www.acul.ohc.cu/b7-9/b7-9-anam.pdf> [Consulta: abril 2012].

GRUPO "LAS CINCO GRACIAS" (2011): "La lengua es un sistema adaptativo complejo". Traducción al español y nota de F. Moreno Fernández. Lingüística em la Red IX <http://www.linred.es/articulos_pdf/LR_articulo_04092011.pdf> [Consulta: abril 2012].

HALLIDAY, M.A.K. (1989): Spoken and Writing language. Oxford: Oxford University Press.

HUNT, K. (1965): Grammatical Structures Written at three grade levels. Urbana: National Council of Teachers of English.

HYMES, D. (1972): On Communicative Competence. Philadelphia: University of Pennsylvania Press. $\quad<\mathrm{http}$ //humanidades.uprrp.edu/smjeg/reserva/Estudios $\% 20 H i s p a n i c o s /$ espa3246/Prof\%20Sunny $\% 20$ Cabrera/ESPA\%203246\%20-

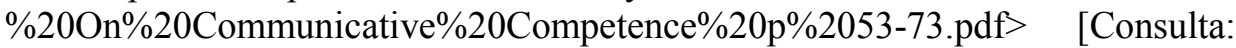
marzo 2012].

HYMES, D. H. (1984): Vers la compétence de communication. Trad. francesa de F. Mugler y nota preliminar de D. Coste. Paris: Hatier CREDIF.

HYMES, D. H. (1985): "Toward Linguistic Competence". Révue de l'AILA/ AILAReview,

$<$ http://www.aila.info/download/publications/review/AILA02.pdf\#page $=7>$ [Consulta: abril 2012].

KARMILOFF-SMITH, A. (1994): Más allá de la modularidad. La ciencia cognitiva desde la perspectiva del desarrollo. Madrid: Alianza editorial.

LÓPEZ MORALES, H. (1984): Enseñanza de la lengua materna. Madrid: Playor.

MASQUELIER, B.y C.TRIMAILLE (2012):"Introduction Dell Hymes: héritages, débats, renouvellements, branchements". Langage et Societé139, 5-19.

OLSON, D. R. (1998): El mundo sobre el papel. El impacto de la escritura y la lectura en la estructura del conocimiento. Barcelona: Gedisa. 
PÉREZ ESTÉVEZ, P. y ZAYAS, F. (2007): Competencia en comunicación lingüistica. Madrid: Alianza editorial.

PÉREZ TAPIA, M.T. (2009): El desarrollo de la competencia comunicativa mediante la reescritura de microrrelatos. Alcalá de Henares: Servicio de Publicaciones de la UAH.

PERINAT, A. (2003): Psicología del desarrollo. Un enfoque sistêmico. Barcelona: Editorial UOC.

SABAH, G. (1995) "Sciences cognitives et conscience". Informe interno LIMSICNRS.

95.17<http://perso.limsi.fr/Individu/gs/textes/Conscience/Conscience.html> [Consulta: febrero 2012].

SANJUÁN BORNAY, N. (2010): "Desarrollando la competencia estratégica y metacognitiva en el aula de ELE mediante el entrenamiento estratégico". Foro Anual de Profesores de ELE VI <http://www.uv.es/foroele/foro6/sanjuan\%20 natalia.pdf $>$ [Consulta: abril 2012].

STEINER, G. y C. Ladjali (2005[2003]): Elogio de la transmisión. Trad. española de G. Cantera. Madrid: Siruela (Biblioteca de Ensayo).

TOLCHINSKY, L. (2011): "La lengua como herramienta de aprendizaje". En U. Ruiz Bikandi (coord.): Lengua Castellana y Literatura. Complementos de formación disciplinar. Barcelona: Graó, 23-41. 